\title{
A Standard Method to Prove That the Riemann Zeta Function Equation Has No Non-Trivial Zeros
}

\author{
Xiaochun Mei \\ Department of Theoretical Physics and Pure Mathematics, Institute of Innovative Physics in Fuzhou, Fuzhou, China \\ Email:ycwlyjs@yeah.net
}

How to cite this paper: Mei, X.C. (2020) A Standard Method to Prove That the Riemann Zeta Function Equation Has No Non-Trivial Zeros. Advances in Pure Mathematics, 10, 86-99.

https://doi.org/10.4236/apm.2020.102006

Received: January 11, 2020

Accepted: February 25, 2020

Published: February 28, 2020

Copyright $\odot 2020$ by author(s) and Scientific Research Publishing Inc. This work is licensed under the Creative Commons Attribution International License (CC BY 4.0).

http://creativecommons.org/licenses/by/4.0/

\section{(c) (i) Open Access}

\begin{abstract}
A standard method is proposed to prove strictly that the Riemann Zeta function equation has no non-trivial zeros. The real part and imaginary part of the Riemann Zeta function equation are separated completely. Suppose $\xi(s)=\xi_{1}(a, b)+i \xi_{2}(a, b)=0$ but $\zeta(s)=\zeta_{1}(a, b)+i \zeta_{2}(a, b) \neq 0$ with $s=a+i b$ at first. By comparing the real part and the imaginary part of Zeta function equation individually, a set of equation about $a$ and $b$ is obtained. It is proved that this equation set only has the solutions of trivial zeros. In order to obtain possible non-trivial zeros, the only way is to suppose that $\zeta_{1}(a, b)=0$ and $\zeta_{2}(a, b)=0$. However, by using the compassion method of infinite series, it is proved that $\zeta_{1}(a, b) \neq 0$ and $\zeta_{2}(a, b) \neq 0$. So the Riemann Zeta function equation has no non-trivial zeros. The Riemann hypothesis does not hold.
\end{abstract}

\section{Keywords}

Riemann Hypothesis, Riemann Zeta Function, Riemann Zeta Function Equation, Jacobi's Function, Residue Theorem, Cauchy-Riemann Equation

\section{Introduction}

In the author's previous paper titled "The inconsistency problem of Riemann Zeta function equation" [1], it was proved that after complex continuation was considered, on the real axis, the Riemann Zeta function equation had serious inconsistency. The Riemann hypothesis was meaningless [1].

In the present discussions of Riemann hypothesis and the calculations of Zeta function's zeros, approximate methods are commonly used. The real part and 
imaginary part of Zeta function equation are mixed together so that problems become complicated. Because the Cauchy-Riemann Equation which an analyzed function should satisfy was violated, the obtained zeros are not the real ones of strict Zeta functions equation [1].

In this paper, a standard method is proposed to separate the real part and the imaginary part of Zeta function equation completely. Then by comparing the real part and the imaginary part individually, it is proved strictly that on whole complex plane, the Zeta function equation has no non-trivial zeros. All trivial zeros are located on the real axis. The Riemann hypothesis is proved untenable again.

The Riemann Zeta function has two forms. One is the form of series summation and another is the form of integral. The form of series summation is more fundamental with

$$
\zeta(s)=\sum_{n=1}^{\infty} n^{-s}, \operatorname{Re}(s)>1
$$

Her $s=a+i b \in C$ is a complex number. Based on Equation (1), by using the Gama function $\Gamma(s)$ and introducing the counter integral on the complex plane, Riemann obtained the integral form of Zeta function, as well as the algebraic relation of Zeta function, called as the Riemann Zeta function Equation [2] [3].

$$
\sum_{n=1}^{\infty} n^{-s}=2(2 \pi)^{s-1} \sin (s \pi / 2) \Gamma(1-s) \sum_{n=1}^{\infty} n^{-(1-s)}
$$

According the definition of Equation (1), we also have

$$
\zeta(1-s)=\sum_{n=1}^{\infty} n^{-(1-s)}
$$

Substituting Equation (1) and Equation (3) in Equation (2), Equation (2) can be written as

$$
\zeta(s)=2(2 \pi)^{s-1} \sin (s \pi / 2) \Gamma(1-s) \zeta(1-s), \operatorname{Re}(s) \neq 1
$$

According to the common understanding at present, on the right side of Equation (4), the definition domain of function is extended from $\operatorname{Re}(s)>1$ to whole complex plane except the point $\operatorname{Re}(s)=1$. So Equation (4) is considered as the new definition of Zeta function after complex continuation. However, by examining Riemann's deduction carefully in his original paper proposed in 1859, we can see that Equation (4) is only a simplified symbol form. The original form of Equation (4) should be Equation (2) [1].

In this original paper, Riemann introduced another form of Zeta function equation [4]

$$
\xi(s)=\frac{1}{2} \pi^{-s / 2} s(s-1) \Gamma(s / 2) \zeta(s)
$$

By using the formula $\theta(x)=\sqrt{x} \theta(1 / x)$ of Jacobi's function, Riemann proved that the function (5) had the symmetry $\xi(s)=\xi(1-s)$. It was considered that 
$\zeta(s)$ described by Equation (4) and $\xi(s)$ described by Equation (5) had the same non-trivial zeros, though they had different forms. In practical discussion, Equation (5) was used to calculate the zeros of Riemann hypothesis in general.

Riemann guessed that all non-trivial zeros were located on the critical line $\operatorname{Re}(s)=1 / 2$ of complex plane, but had not provided any concrete zero. Over one hundred years, mathematicians had done a lot of research on the Riemann hypothesis, trying to prove or falsify it, but nothing worked. The Riemann hypothesis becomes the world mathematics problem.

However, there is a third possibility, i.e., there is something wrong with the Riemann Zeta function equation itself, so that the Riemann hypothesis can not be proved. It was proved in the author's previous paper that there were four basic mistakes in the Riemann's original paper in 1859, the Riemann Zeta function did not hold, the Riemann hypothesis becomes meaningless [1].

1) An integral item around the original point of coordinate system was neglected in Riemann's original paper. The item was convergent when $\operatorname{Re}(s)>1$, but infinite when $\operatorname{Re}(s) \leq 1$. That is to say, the integral form of Riemann Zeta function has not changed its divergence of series summation form. The Riemann Zeta function Equations (2) and (4) do not hold.

2) The Riemann Zeta function equation has serious inconsistency. The so-called continuation of function indicates that a function which has no meaning in a certain domain is re-defined so that it becomes meaningful in this domain. But there is a basic requirement for the function's continuation, i.e., this new defined function should have the same form with original function in the original domain. Otherwise, the extended function can not be regarded as the continuation of original function [5]. According to this basic principle, in the domain of $\operatorname{Re}(s)>1$, the left side of Equation (4) should be in the form of Equation (1).

On the other hand, Equation (4) has definition on whole complex plane except the point $\operatorname{Re}(s)=1$ i.e., $a \neq 1$ but $b$ can be arbitrary. Taking $a=3.5$ and $b=0$, Equation (4) should be effective. Because the original form of Equation (4) is Equation (2), it means that Equation (2) should be effective on $a=3.5$ and $b=0$. However, it is proved that the left side of Equation (2) is a limited value but the right side of Equation (2) is infinite when $a=3.5$ and $b=0$, so the two sides of Riemann Zeta function equation are inconsistent [1].

In fact, it is proved that on the real axis, the Riemann Zeta function equation only holds at the point $s=a=1 / 2$. However, at this point, the Zeta function is infinite, rather than zero. At the other points of real axis, if the left side of Equation (2) is convergent, the right side of Equation (2) is divergent, and vice versa. So the two sides of the Riemann Zeta function equation are incompatible.

3) A summation formula was used in the deduction of the integral form of Riemann zeta function. The applicable condition of this formula is $x>0$. At point $x=0$, the formula becomes meaningless. However, the lower limit of Zeta function integral is $x=0$, so this formula can not be used. The integral form of Riemann zeta function does not hold. 
4) The formula $\theta(x)=\sqrt{x} \theta(1 / x)$ of Jacobi function was used to prove the symmetry of Zeta function. The applicable condition of this formula is $x>0$ [4]. But the lower limit of integral involved in the deduction is $x=0$. Therefore, the formula can not be used too, the symmetry $\xi(s)=\xi(1-s)$ does not hold.

The zeros calculation of Riemann Zeta function were discussed in the paper [1]. At present, it has been proved by manual and computer numerical methods that there are lot of zeros on the critical line of $a=1 / 2$. The number has exceeded 10 trillion [6]. The paper pointed out that all methods used in the calculations were approximate ones. For example, Equation (5) was developed into the infinite series called as the Riemann-Siegal formula, then the zero of each polynomial formula was calculated. The result violated the symmetry of the Cauchy-Riemann formula that any analytic functions should satisfy, so they were not the true zeros of strict Zeta functions.

In this paper, regardless of these problems mentioned above, we suppose that the Riemann Zeta function equation still holds and discuss the zero problem of Zeta function strictly. A simple and standard method is proposed to prove that the Riemann Zeta function equation has no non-trivial zeros on whole complex plane.

Let $\xi=\xi_{1}+i \xi_{2}$ and $\zeta=\zeta_{1}+i \zeta_{2}$, by separating each item of Equation (5) into real and imaginary parts, Equation (5) is written as the forms that real part and imaginary part are separated completely. Then we discuss the zeros of real part and imaginary part individually.

At first, suppose that $\xi_{1}=0$ and $\xi_{2}=0$, but $\zeta_{1} \neq 0$ and $\zeta_{2} \neq 0$, we obtain a set of equation about $a$ and $b$. It is proved that the only solution to this equation set is $a=1$ and $b=0$. But they are the trivial zeros located on the real axis, not non-trivial zeros. So Equation (5) has no non-trivial zeros. By the same method, it also is proved that Equation (4) only has no trivial zeros which are located at the points $a=-2 n \quad(n=0,1,2, \cdots)$ and $b=0$.

At last, in order to obtain possible non-trivial zeros, we take $\zeta_{1}=0$ and $\zeta_{2}=0$, i.e., the summation form of Zeta function itself is equal to zero. However, by using the compassion method of infinite series, it is proved that $\zeta_{1}$ and $\zeta_{2}$ can not be zeros simultaneously.

Therefore, we prove that the Riemann Zeta function equation has no non-trivial zeros again, the Riemann hypothesis does not hold.

\section{The Proof That the Zeta Function Equation (5) Has No Non-Trivial Zeros}

We discuss the zeros of Equation (5) in this section. Then discuss the zeros of Equation (4) in next section.

Theorem 1. On the complex plane, if the real part and imaginary part of zeta function $\zeta(s)$ are not equal to zeros, the Zeta function equation (5) has no non-trivial zeros. The trivial zero is located on the real axis at the point $a=1$ and $b=0$. 
Proof: We separate the real part and imaginary part of $\xi(s)$ and $\zeta(s)$, write them as

$$
\begin{aligned}
& \xi(s)=\xi_{1}(a, b)+i \xi_{2}(a, b) \\
& \zeta(s)=\zeta_{1}(a, b)+i \zeta_{2}(a, b)
\end{aligned}
$$

Here $\xi_{1}, \xi_{2}, \zeta_{1}$ and $\zeta_{2}$ are real functions. By using formula $t=\mathrm{e}^{\ln t}$, we have

$$
\begin{gathered}
\pi^{-s / 2}=\pi^{-(a+i b) / 2}=\pi^{-a / 2} \pi^{-i b / 2} \\
=\pi^{-a / 2}\left(\mathrm{e}^{\ln \pi}\right)^{-i b / 2}=\pi^{-a / 2} \mathrm{e}^{-i(b \ln \pi) / 2} \\
=\pi^{-a / 2}[\cos [(b \ln \pi) / 2]-i \sin [(b \ln \pi) / 2]] \\
s(s-1)=(a+i b)(a-1+i b)=a(a-1)-b^{2}+i(2 a b-b) \\
\frac{1}{2} \pi^{-s / 2} s(s-1) \\
=\frac{1}{2} \pi^{-a / 2}\left[\left[a(a-1)-b^{2}\right] \cos [(b \ln \pi) / 2]+(2 a b-b) \sin [(b \ln \pi) / 2]\right] \\
+i \frac{1}{2} \pi^{-a / 2}\left[(2 a b-b) \cos [(b \ln \pi) / 2]-\left[a(a-1)-b^{2}\right] \sin [(b \ln \pi) / 2]\right]
\end{gathered}
$$

Let

$G_{1}=\frac{1}{2} \pi^{-a / 2}\left[\left[a(a-1)-b^{2}\right] \cos [(b \ln \pi) / 2]+(2 a b-b) \sin [(b \ln \pi) / 2]\right]$

$G_{2}=\frac{1}{2} \pi^{-a / 2}\left[(2 a b-b) \cos [(b \ln \pi) / 2]-\left[a(a-1)-b^{2}\right] \sin [(b \ln \pi) / 2]\right]$

We get

$$
\frac{1}{2} \pi^{-s / 2} s(s-1)=G_{1}+i G_{2}
$$

Here $G_{1}$ and $G_{2}$ are real functions. On the other hand, the definition of real Gama function is [6]

$$
\Gamma(a)=\int_{0}^{\infty} \mathrm{e}^{-t} t^{a-1} \mathrm{~d} t>0, a>0
$$

Let $a \rightarrow s=a+i b$, we obtain the complex continuation of Gama function. We have

$$
\begin{aligned}
\Gamma(s / 2)= & \int_{0}^{\infty} \mathrm{e}^{-t} t^{s / 2-1} \mathrm{~d} t=\int_{0}^{\infty} \mathrm{e}^{-t} t^{a / 2-1} t^{i b / 2} \mathrm{~d} t=\int_{0}^{\infty} \mathrm{e}^{-t} t^{a / 2-1} \mathrm{e}^{i(b \ln t) / 2} \mathrm{~d} t \\
= & \int_{0}^{\infty} \mathrm{e}^{-t} t^{a / 2-1}(\cos [(b \ln t) / 2]+i \sin [(b \ln t) / 2]) \mathrm{d} t \\
= & \Gamma_{1}(a, b)+i \Gamma_{2}(a, b) \\
& \Gamma_{1}(a, b)=\int_{0}^{\infty} \mathrm{e}^{-t} t^{a / 2-1} \cos [(b \ln t) / 2] \mathrm{d} t
\end{aligned}
$$




$$
\Gamma_{2}(a, b)=\int_{0}^{\infty} \mathrm{e}^{-t} t^{a / 2-1} \sin [(b \ln t) / 2] \mathrm{d} t
$$

$\Gamma_{1}$ and $\Gamma_{2}$ are also real functions. Therefore, according to the equations above, Equation (5) can be written as

$$
\begin{aligned}
\xi_{1}+i \xi_{2}= & \left(G_{1}+i G_{2}\right)\left(\Gamma_{1}+i \Gamma_{2}\right)\left(\zeta_{1}+i \zeta_{2}\right) \\
= & \left(G_{1} \Gamma_{1}-G_{2} \Gamma_{2}\right) \zeta_{1}-\left(G_{1} \Gamma_{2}+G_{2} \Gamma_{1}\right) \zeta_{2} \\
& +i\left[\left(G_{1} \Gamma_{2}+G_{2} \Gamma_{1}\right) \zeta_{1}+\left(G_{1} \Gamma_{1}-G_{2} \Gamma_{2}\right) \zeta_{2}\right]
\end{aligned}
$$

The real part and imaginary part of Equation (16) are separated with

$$
\begin{aligned}
& \xi_{1}=\left(G_{1} \Gamma_{1}-G_{2} \Gamma_{2}\right) \zeta_{1}-\left(G_{1} \Gamma_{2}+G_{2} \Gamma_{1}\right) \zeta_{2} \\
& \xi_{2}=\left(G_{1} \Gamma_{2}+G_{2} \Gamma_{1}\right) \zeta_{1}+\left(G_{1} \Gamma_{1}-G_{2} \Gamma_{2}\right) \zeta_{2}
\end{aligned}
$$

If the Zeta function equation has zeros, its real part and imaginary part should be equal to zero simultaneously. Let $\xi_{1}=0$ and $\xi_{2}=0$, we obtain

$$
\begin{aligned}
& \left(G_{1} \Gamma_{1}-G_{2} \Gamma_{2}\right) \zeta_{1}-\left(G_{1} \Gamma_{2}+G_{2} \Gamma_{1}\right) \zeta_{2}=0 \\
& \left(G_{1} \Gamma_{2}+G_{2} \Gamma_{1}\right) \zeta_{1}+\left(G_{1} \Gamma_{1}-G_{2} \Gamma_{2}\right) \zeta_{2}=0
\end{aligned}
$$

If $\zeta_{1} \neq 0$ and $\zeta_{2} \neq 0$, we can obtain from Equation (19)

$$
\zeta_{1}=\frac{G_{1} \Gamma_{2}+G_{2} \Gamma_{1}}{G_{1} \Gamma_{1}-G_{2} \Gamma_{2}} \zeta_{2}
$$

Substitute Equation (21) in Equation (20), we get

$$
\frac{\left(G_{1} \Gamma_{2}+G_{2} \Gamma_{1}\right)^{2}}{G_{1} \Gamma_{1}-G_{2} \Gamma_{2}} \zeta_{2}+\left(G_{1} \Gamma_{1}-G_{2} \Gamma_{2}\right) \zeta_{2}=0
$$

or

$$
\left(G_{1} \Gamma_{2}+G_{2} \Gamma_{1}\right)^{2}+\left(G_{1} \Gamma_{1}-G_{2} \Gamma_{2}\right)^{2}=0
$$

Because it is the square summation of two items, each one in Equation (23) should be zero simultaneously

$$
\begin{aligned}
& G_{1} \Gamma_{2}+G_{2} \Gamma_{1}=0 \\
& G_{1} \Gamma_{1}-G_{2} \Gamma_{2}=0
\end{aligned}
$$

From Equation (25), we have $\Gamma_{1}=G_{2} \Gamma_{2} / G_{1}$. Substitute it in Equation (24), we get $G_{1}^{2}+G_{2}^{2}=0$. Because $G_{1}^{2}$ and $G_{2}^{2}$ can not be negative, we can only have $G_{1}=0$ and $G_{2}=0$. According to Equation (11), the results are

$$
\begin{aligned}
& {\left[a(a-1)-b^{2}\right] \cos [(b \ln \pi) / 2]+(2 a b-b) \sin [(b \ln \pi) / 2]=0} \\
& -\left[a(a-1)-b^{2}\right] \sin [(b \ln \pi) / 2]+(2 a b-b) \cos [(b \ln \pi) / 2]=0
\end{aligned}
$$

To square them and add them together, we get

$$
\left[a(a-1)-b^{2}\right]^{2}+(2 a b-b)^{2}=0
$$

Equation (28) indicates $a(a-1)-b^{2}=0$ and $2 a b-b=0$. Due to that $a$ and $b$ are real numbers, the solutions of these two formulas are $a=0,1$ and $b=0$. Obviously, they are trivial zeros located on the real axis. In fact, because 
of $\pi^{-s / 2} \neq 0$ this solution is the result of $s(s-1)=0$ in Equation (9). Let its real part and imaginary part be equal to zeros simultaneously, we have $a(a-1)-b^{2}=0$ and $2 a b-b=0$. Obviously, the value $a=1 / 2$ of Riemann's hypothesis is not the solution of Equation (28).

The result above has nothing to do with the $\Gamma(s)$ function. We should consider the zeros of $\Gamma(s)$ function. It is obvious that when $b \neq 0, \Gamma_{1}(a, b)$ and $\Gamma_{2}(a, b)$ described by Equation (15) are not equal to each other. So they can not be equal to zeros simultaneously (if they have zeros). Similar to the real $\Gamma(a)$ function, it indicates that the $\Gamma(s)$ function of complex continuation has no zeros too.

As we known that when $b=0$ and $a / 2>0$ or $a>0, \Gamma(a / 2)$ is limited but not equal to zero. When $a / 2=0,-1,-2, \cdots$, or $a=0,-2,-4, \cdots, \Gamma(a / 2)$ is infinite [5]. Therefore, after $\Gamma(s / 2)$ function is considered, the zero $a=0$ in Equation (28) is canceled. The trivial zero of Zeta function Equation (5) is located at $a=1$ and $b=0$. Thus, the proof of Theorem 1 is finished.

Besides, if we want to look for the non-trivial zeros of $\xi(s)$, the last way is to let $\zeta_{1}=0$ and $\zeta_{2}=0$ in which the non-trivial zeros may be contained. In this case, the problem whether or not the series summation form of Zeta function can be equal to zero is involved. We will discuss this problem in Section 4.

\section{The Proof That the Zeta Function Equation (4) Has No Non-Trivial Zeros}

Theorem 2. On the complex plane, if the real part and imaginary part of Zeta function $\zeta(1-s)$ are not equal to zeros, the Zeta function equation Equation (4) has no non-trivial zeros. The trivial zeros are located on the real axis at the points $a=-2 n \quad(n=0,1,2, \cdots)$ and $b=0$.

Proof: Let $\zeta(s) \rightarrow \zeta^{\prime}(s)$ and write Equation (4) as

$$
\zeta^{\prime}(s)=2(2 \pi)^{s-1} \sin (s \pi / 2) \Gamma(1-s) \zeta(1-s)
$$

According to current understanding, $\zeta^{\prime}(s)$ is considered as the new definition of Zeta function after complex continuation was carried out. But $\zeta(1-s)$ still has the same form of Equation (1), because Equation (3) was used in the last steep of Riemann's deduction. By using the formula $i=\mathrm{e}^{i \pi / 2}$, we have

$$
\begin{aligned}
& \sin (s \pi / 2)=\frac{\mathrm{e}^{i s \pi / 2}-\mathrm{e}^{-i s \pi / 2}}{2 i}=\frac{\mathrm{e}^{-i \pi / 2}}{2}\left[\mathrm{e}^{i(a+i b) \pi / 2}-\mathrm{e}^{-i(a+i b) \pi / 2}\right] \\
& =\frac{1}{2}\left[\mathrm{e}^{-b \pi / 2} \mathrm{e}^{i(a-1) \pi / 2}-\mathrm{e}^{b \pi / 2} \mathrm{e}^{-i(a+1) \pi / 2}\right] \\
& 2(2 \pi)^{s-1}=2(2 \pi)^{a-1+i b}=2(2 \pi)^{a-1}(2 \pi)^{i b}=2(2 \pi)^{a-1} \mathrm{e}^{i b \ln 2 \pi} \\
& 2(2 \pi)^{s-1} \sin (s \pi / 2) \\
& =(2 \pi)^{a-1} \mathrm{e}^{i b \ln 2 \pi}\left[\mathrm{e}^{-b \pi / 2} \mathrm{e}^{i(a-1) \pi / 2}-\mathrm{e}^{b \pi / 2} \mathrm{e}^{-i(a+1) \pi / 2}\right] \\
& =(2 \pi)^{a-1}\left[\mathrm{e}^{-b \pi / 2} \mathrm{e}^{i[(a-1) \pi / 2+b \ln 2 \pi]}-\mathrm{e}^{b \pi / 2} \mathrm{e}^{-i[(a+1) \pi / 2-b \ln 2 \pi]}\right]
\end{aligned}
$$

Let 


$$
A=(a-1) \pi / 2+b \ln 2 \pi, B=(a+1) \pi / 2-b \ln 2 \pi
$$

Equation (32) can be written as

$$
\begin{gathered}
2(2 \pi)^{s-1} \sin (s \pi / 2)=G_{1}+i G_{2} \\
=(2 \pi)^{a-1}\left[\mathrm{e}^{-b \pi / 2} \cos A-\mathrm{e}^{b \pi / 2} \cos B+i\left(\mathrm{e}^{-b \pi / 2} \sin A+\mathrm{e}^{b \pi / 2} \sin B\right)\right] \\
G_{1}=(2 \pi)^{a-1}\left(\mathrm{e}^{-b \pi / 2} \cos A-\mathrm{e}^{b \pi / 2} \cos B\right) \\
G_{2}=(2 \pi)^{a-1}\left(\mathrm{e}^{-b \pi / 2} \sin A+\mathrm{e}^{b \pi / 2} \sin B\right)
\end{gathered}
$$

Here $A, B, G_{1}$ and $G_{2}$ are real functions. We also have [6]

$$
\begin{gathered}
\Gamma(1-s)=\int_{0}^{\infty} \mathrm{e}^{-t} t^{1-s-1} \mathrm{~d} t=\int_{0}^{\infty} \mathrm{e}^{-t} t^{-a} t^{-i b} \mathrm{~d} t=\int_{0}^{\infty} \mathrm{e}^{-t} t^{-a} \mathrm{e}^{-i b \ln t} \mathrm{~d} t \\
=\int_{0}^{\infty} \mathrm{e}^{-t} t^{1-a}(\cos (b \ln t)-i \sin (b \ln t)) \mathrm{d} t \\
=\Gamma_{1}(a, b)+i \Gamma_{2}(a, b)
\end{gathered}
$$

Here $\Gamma_{1}$ and $\Gamma_{2}$ are also real functions. Thus, Equation (4) can be written as

$$
\begin{aligned}
\zeta^{\prime}= & \zeta_{1}^{\prime}+i \zeta_{2}^{\prime}=\left(G_{1}+i G_{2}\right)\left(\Gamma_{1}+i \Gamma_{2}\right)\left(\zeta_{1}+i \zeta_{2}\right) \\
= & \left(G_{1} \Gamma_{1}-G_{2} \Gamma_{2}\right) \zeta_{1}-\left(G_{1} \Gamma_{2}+G_{2} \Gamma_{1}\right) \zeta_{2} \\
& +i\left[\left(G_{1} \Gamma_{2}+G_{2} \Gamma_{1}\right) \zeta_{1}+\left(G_{1} \Gamma_{1}-G_{2} \Gamma_{2}\right) \zeta_{2}\right]
\end{aligned}
$$

By separating real part and imaginary part, we get

$$
\begin{aligned}
& \zeta_{1}^{\prime}=\left(G_{1} \Gamma_{1}-G_{2} \Gamma_{2}\right) \zeta_{1}-\left(G_{1} \Gamma_{2}+G_{2} \Gamma_{1}\right) \zeta_{2} \\
& \zeta_{2}^{\prime}=\left(G_{1} \Gamma_{2}+G_{2} \Gamma_{1}\right) \zeta_{1}+\left(G_{1} \Gamma_{1}-G_{2} \Gamma_{2}\right) \zeta_{2}
\end{aligned}
$$

If the Zeta function equation has zeros, its real part and imaginary part should be zeros simultaneously. Suppose that $\zeta_{1} \neq 0$ and $\zeta_{2} \neq 0$, according to the same method as shown in Section 2, we get

$$
\begin{aligned}
& G_{1} \Gamma_{2}+G_{2} \Gamma_{1}=0 \\
& G_{1} \Gamma_{1}-G_{2} \Gamma_{2}=0
\end{aligned}
$$

Form Equation (42), we have $\Gamma_{1}=G_{2} \Gamma_{2} / G_{1}$. Substituting it in Equation (41), we get $G_{1}^{2}+G_{2}^{2}=0$. The only solutions are $G_{1}=0$ and $G_{2}=0$. According to Equation (35), we obtain

$$
\begin{aligned}
& \mathrm{e}^{-b \pi / 2} \cos A-\mathrm{e}^{b \pi / 2} \cos B=0 \\
& \mathrm{e}^{-b \pi / 2} \sin A+\mathrm{e}^{b \pi / 2} \sin B=0
\end{aligned}
$$

To square Equation (43) and Equation (44), then add them together, we get

$$
\mathrm{e}^{-b \pi}+\mathrm{e}^{b \pi}=2 \cos (A+B)
$$

To square Equation (43) and Equation (44) and subtract them, we get 


$$
\mathrm{e}^{-b \pi} \cos 2 A+\mathrm{e}^{b \pi} \cos 2 B=2 \cos (A-B)
$$

Substituting Equation (33) in Equation (45) and Equation (46), we obtain

$$
\begin{gathered}
\mathrm{e}^{-b \pi}+\mathrm{e}^{b \pi}=2 \cos (a \pi) \\
-\mathrm{e}^{-b \pi} \cos (a \pi+2 b \ln 2 \pi)-\mathrm{e}^{b \pi} \cos (a \pi-2 b \ln 2 \pi)=-2 \cos (2 b \ln 2 \pi)
\end{gathered}
$$

If $b \neq 0$, by developing $\mathrm{e}^{-b \pi}$ and $\mathrm{e}^{b \pi}$ into the series, we can prove $\mathrm{e}^{-b \pi}+\mathrm{e}^{b \pi}>2$. However, because of $2 \cos (a \pi) \leq 2$, Equation (47) does not hold. So the only solution of Equation (47) is $b=0$ and $a= \pm 2 n(n=0,1,2, \cdots)$ representing the trivial zeros located on the real axis. In this case, Equations (47) and (48) become the same with the same solution.

If taking $a=1 / 2$, we have $2 \cos (\pi / 2)=0$. Because of $\mathrm{e}^{-b \pi}+\mathrm{e}^{b \pi} \neq 0$, Equation (47) can not hold too. Therefore, $a=1 / 2$ is not the solution of Equation (4) too. In fact, because of $2 \pi^{\text {is }} \neq 0$, the solutions $b=0$ and $a= \pm 2 n$ are the result of $\sin (s \pi / 2)=0$ in Equation (29), having nothing to do with $\Gamma$ function too.

But if let $b=0$ and $-a=a^{\prime}-1$ in Equation (37), we have $a^{\prime}=1-a$. When $a^{\prime} \leq 0$ or $a \geq 1$, we have $\Gamma_{1} \rightarrow \infty$. Therefore, the corresponding zeros $a=2,4,6, \cdots$ in (47) can be removed. The trivial zeros of Equation (4) only appear at the points $a=-2 n \quad(n=0,1,2, \cdots)$ and $b=0$. Thus, the proof of Theorem 2 is finished.

So, looking for possible non-trivial zeros of Equation (4), the only way for us is to consider $\zeta_{1}(a, b)=0$ and $\zeta_{2}(a, b)=0$. The problem is involved whether or not the series summation form of Zeta function can be equal to zero. According to Equation (1), we have

$$
\begin{aligned}
\zeta(1-s)= & \zeta_{1}(a, b)+i \zeta_{2}(a, b) \\
= & 1+\frac{1}{2^{1-s}}+\frac{1}{3^{1-s}}+\cdots+\frac{1}{n^{1-s}}+\cdots \\
= & 1+2^{a-1} \mathrm{e}^{i b \ln 2}+3^{a-1} \mathrm{e}^{i b \ln 3}+\cdots+n^{a-1} \mathrm{e}^{i b \ln n}+\cdots \\
= & 1+2^{a-1} \cos (b \ln 2)+3^{a-1} \cos (b \ln 3)+\cdots+n^{a-1} \cos (b \ln n)+\cdots \\
& +i\left(2^{a-1} \sin (b \ln 2)+3^{a-1} \sin (b \ln 3)+\cdots+n^{a-1} \sin (b \ln n)+\cdots\right)
\end{aligned}
$$

So we obtain

$$
\begin{gathered}
\zeta_{1}(a, b)=1+2^{a-1} \cos (b \ln 2)+3^{a-1} \cos (b \ln 3)+\cdots+n^{a-1} \cos (b \ln n)+\cdots \\
\zeta_{2}(a, b)=2^{a-1} \sin (b \ln 2)+3^{a-1} \sin (b \ln 3)+\cdots+n^{a-1} \sin (b \ln n)+\cdots
\end{gathered}
$$

We discuss the zeros of Equations (50) and (51) in next section.

\section{The Proof That the Series Summation Formula of Riemann Zeta Function Has No Zeros}

\subsection{The Convergence of Summation Form of Zeta Function}

In order to discuss the zeros of the summation form of Zeta function, we should discuss its convergence. If $s=a$ is a real number, Equation (1) is divergent 
when $a<1$ without zeros. When $a>1$, the series is convergent and great than zero, so (1) has no zeros. The proof is as below [7].

Suppose $a>1, p$ is prime number, by considering the Euler prime number product formula

$$
|\zeta(a)|=\prod_{p}\left|1-p^{-a}\right|^{-1} \geq \prod_{p}\left|1+p^{-a}\right|^{-1}=\exp \left[-\sum_{p} \ln \left(1+p^{-a}\right)\right]
$$

For any $x>0$, we have $\ln (1+x)<x$. Because $\sum p^{-a}<\infty$ is convergent, we have

$$
|\zeta(a)| \geq \exp \left[-\sum_{p} p^{-a}\right]>0
$$

If $s=a+i b$ is a complex number, the situation is more complicated. It does not seems to have strict proof to conform $\zeta(s) \neq 0$ at present. Let's discuss this problem below. According to the judgment formula of series convergence of complex function showing in Equation (1), we have

$$
\lim _{n \rightarrow \infty}\left|\frac{u_{n+1}}{u_{n}}\right|=\lim _{n \rightarrow \infty}\left|\frac{n^{a+i b}}{(n+1)^{a+i b}}\right|=\lim _{n \rightarrow \infty}\left|\left(\frac{1}{1+1 / n}\right)^{a+i b}\right|=1
$$

Because the radius of convergence is 1, we can not judge the convergence of Equation (1). By using the Euler formula, we write Equation (1) as

$$
\begin{aligned}
\zeta(s)= & 1+\frac{1}{2^{a+i b}}+\frac{1}{3^{a+i b}}+\cdots+\frac{1}{n^{a+i b}}+\cdots \\
= & 1+2^{-a} \cos (b \ln 2)+3^{-a} \cos (b \ln 3)+\cdots+n^{-a} \cos (b \ln n)+\cdots \\
& -i\left(2^{-a} \sin (b \ln 2)+3^{-a} \sin (b \ln 3)+\cdots+n^{-a} \sin (b \ln n)+\cdots\right)
\end{aligned}
$$

By separating the real part and imaginary part, let $\zeta(s)=u+i v$, we get

$$
\begin{aligned}
& u(a, b)=1+2^{-a} \cos (b \ln 2)+3^{-a \cdot} \cos (b \ln 3)+\cdots+n^{-a} \cos (b \ln n)+\cdots \\
& v(a, b)=-\left(2^{-a} \sin (b \ln 2)+3^{-a} \sin (b \ln 3)+\cdots+n^{-a} \sin (b \ln n)+\cdots\right)
\end{aligned}
$$

By using the formula $\ln (n+1)=\ln n+\ln (1+1 / n)$ and considering the convergences of Equations (56) and (57), we have:

$$
\begin{aligned}
& \lim _{n \rightarrow \infty} \frac{u_{n+1}}{u_{n}}=\lim _{n \rightarrow \infty} \frac{n^{a} \cos (b \ln (n+1))}{(n+1)^{a} \cos (b \ln n)} \\
&=\lim _{n \rightarrow \infty} \frac{\cos (b \ln n+b \ln (1+1 / n))}{(1+1 / n)^{a} \cos (b \ln n)} \\
&=\lim _{n \rightarrow \infty} \frac{\cos (b \ln n)}{\cos (b \ln n)}=1 \\
& \lim _{n \rightarrow \infty} \frac{v_{n+1}}{v_{n}}=\lim _{n \rightarrow \infty} \frac{n^{a} \sin (b \ln (n+1))}{(n+1)^{a} \sin (b \ln n)}=\lim _{n \rightarrow \infty} \frac{\sin (b \ln n)}{\sin (b \ln n)}=1
\end{aligned}
$$

The radius of convergence is still equal to 1. The convergences of Equation (56) and Equation (57) can not be determined. Because the formulas contain 
Trigonometric functions, the items in the formula can be positive or negative, we can not ensure that the result of summation is always great than zero. This is different from the situation when $s$ is a real number.

\subsection{The Zeros of Common Analytic Functions}

In the theory of complex functions, the analytic nature of functions is very important. Many theorems cannot be used for non-analytic function. For example, the residue theorem is effective only for analytic functions. On the other hand, a complex function can always be written as

$$
f(z)=u(x, y)+i v(x, y)
$$

Here $z=x+i y$. If $f(z)$ is analytic one, its real part and imaginary part are related. The Cauchy-Riemann formula should be satisfied with [6]

$$
\frac{\partial u}{\partial x}=\frac{\partial v}{\partial y}, \frac{\partial u}{\partial y}=-\frac{\partial v}{\partial x}
$$

In the current calculation of the zero point of Riemann Zeta function, some approximate methods are adopted. Because Equation (61) is ignored, what obtained are not real zeros of Zeta function [1].

It should be emphasized that when we calculate the zero points of analytic functions, we need to separate the real part and the imaginary part. Because it's possible to have a situation where the real part or the imaginary part is equal to zero, but they are not equal to zero simultaneously. However, in the current zero calculation of Riemann hypothesis, the real part and imaginary part are often mixed together, making the problem ambiguous.

\subsection{The Proof That the Series Summation Formula of Riemann Zeta Function Has No Zeros on the Complex Plane}

Theorem 3. The series summation formula of Riemann Zeta function has no zeros on whole complex plane.

Proof: By using the formula $n^{a}=\mathrm{e}^{a \ln n}$, we write Equations (50) and (51) as

$$
\begin{array}{r}
\zeta_{1}(a, b)=u(a, b)=1+\mathrm{e}^{(a-1) \ln 2} \cos (b \ln 2)+\mathrm{e}^{(a-1) \ln 3} \cos (b \ln 3)+\cdots \\
\zeta_{2}(a, b)=v(a, b)=\mathrm{e}^{(a-1) \ln 2} \sin (b \ln 2)+\mathrm{e}^{(a-1) \ln 3} \sin (b \ln 3)+\cdots
\end{array}
$$

It is easy to prove that Equations (62) and (63) satisfy Equation (61). So the summation form of Zeta function is an analytic one. We prove below that Equations (62) and (63) can not be equal to zero simultaneously.

Let's discuss the simplest situation to take the first two items in $\zeta(1-s)=0$ as shown in Equation (49). According to Equations (62) and (63), we have

$$
\begin{gathered}
1+\mathrm{e}^{(a-1) \ln 2} \cos (b \ln 2)=0 \\
\mathrm{e}^{(a-1)} \sin (b \ln 2)=0
\end{gathered}
$$

Suppose that $a$ is a limited number, the solution of Equation (65) is $b \ln 2=n \pi$. If $n$ is an even number, we have $\cos n \pi=1$. Substituting it in Equa- 
tion (64), we get

$$
1+\mathrm{e}^{(a-1) \ln 2}=0 \text { or }(a-1) \ln 2=\ln (-1)
$$

Because $a$ is a real number, $\ln (-1)=\ln \mathrm{e}^{i \pi}=i \pi$ is not a real number, so Equation (64) has no solution of real number. When $n$ is an odd number, we have $\cos n \pi=-1$. Substituting it in Equation (64), we get

$$
1-\mathrm{e}^{(a-1) \ln 2}=0 \text { or }(a-1) \ln 2=\ln 1=0
$$

Therefore, the solutions of Equations (64) and (65) are $a=1$ and $b=(2 n+1) \pi / \ln 2 \quad(n=0,1,2, \cdots)$. If $a \neq 1$, Equations (64) and (65) can not be equal to zeros simultaneously.

Then, let's discuss the first three items of Equation (49). By considering $n^{a}=\mathrm{e}^{a \ln n}$ and $n^{i b}=\mathrm{e}^{i b \ln n}$, multiplying the first three items with $2^{(1-s)} 3^{(1-s)}=(2 \cdot 3)^{(1-a)} \mathrm{e}^{-i b \ln (2 \cdot 3)}$, and let it equal to zero, we get

$$
(2 \cdot 3)^{(1-a)} \mathrm{e}^{-i b \ln (2 \cdot 3)}+2^{(1-a)} \mathrm{e}^{-i b \ln 2}+3^{(1-a)} \mathrm{e}^{-i b \ln 3}=0
$$

By considering the Euler's formula and separating real part and imaginary part, we obtain

$$
\begin{aligned}
& (2 \cdot 3)^{(1-a)} \cos (b \ln (2 \cdot 3))+2^{(1-a)} \cos (b \ln 2)+3^{(1-a)} \cos (b \ln 3)=0 \\
& (2 \cdot 3)^{(1-a)} \sin (b \ln (2 \cdot 3))+2^{(1-a)} \sin (b \ln 2)+3^{(1-a)} \sin (b \ln 3)=0
\end{aligned}
$$

We write Equation (70) as

$$
(2 \cdot 3)^{(1-a)} \cos \left(\frac{\pi}{2}-b \ln (2 \cdot 3)\right)+2^{(1-a)} \cos \left(\frac{\pi}{2}-b \ln 2\right)+3^{(1-a)} \cos \left(\frac{\pi}{2}-b \ln 3\right)=0
$$

It can be seen that Equations (69) and (71) are completely symmetric with the same parameters before cosine function. Because $a$ and $b$ are arbitrary, in order to make Equations (69) and (71) tenable for arbitrary $a$ and $b$, the only way is to let

$$
b \ln (2 \cdot 3)=\frac{\pi}{2}-b \ln (2 \cdot 3), b \ln 2=\frac{\pi}{2}-b \ln 2, b \ln 3=\frac{\pi}{2}-b \ln 3
$$

or

$$
b=\frac{\pi}{4 \ln (2 \cdot 3)}, b=\frac{\pi}{4 \ln 2}, b=\frac{\pi}{4 \ln 3}
$$

However, these three relations are contradictory. So Equations (69) and (70) can not be equal to zero simultaneously.

Off cause, to the series of which item's number is limited, the proof above is not strict. But for the series with infinite items, this method is standard one. Let

$$
\zeta(1-s)=1+\frac{1}{2^{1-s}}+\frac{1}{3^{1-s}}+\cdots+\frac{1}{p_{n}^{1-s}}+\cdots=0
$$

by multiplying two sides of Equation (74) with

$$
\left(2 \cdot 3 \cdots p_{n}\right)^{(1-s)}\left(p_{2} p_{3} \cdots p_{n-1} p_{n}\right)^{(1-a)} \mathrm{e}^{-i b \ln \left(p_{2} p_{3} \cdots p_{n-1} p_{n}\right)} \text {, we get }
$$




$$
\begin{aligned}
& \left(p_{2} p_{3} \cdots p_{n-1}\right)^{(1-a)} \mathrm{e}^{-i b \ln \left(p_{2} p_{3} \cdots p_{n-1}\right)}+\left(p_{2} p_{3} \cdots p_{n-2} p_{n}\right)^{(1-a)} \mathrm{e}^{-i b \ln \left(p_{2} p_{3} \cdots p_{n-2} p_{n}\right)}+\cdots \\
& +\left(p_{3} \cdots p_{n-1} p_{n}\right)^{(1-a)} \mathrm{e}^{-i b \ln \left(p_{2} p_{3} \cdots p_{n-1}\right)}+\left(p_{2} p_{3} \cdots p_{n-1} p_{n}\right)^{(1-a)} \mathrm{e}^{-i b \ln \left(p_{2} p_{3} \cdots p_{n-1} p_{n}\right)}=0
\end{aligned}
$$

For infinite series, we have $p_{n} \rightarrow \infty$. By dividing real part and imaginary part, similar to Equations (69) and (71), we obtain

$$
\begin{aligned}
& \left(p_{2} p_{3} \cdots p_{n-1}\right)^{(1-a)} \cos \left(b \ln \left(p_{2} p_{3} \cdots p_{n-1}\right)\right) \\
& +\left(p_{2} p_{3} \cdots p_{n-2} p_{n}\right)^{(1-a)} \cos \left(b \ln \left(p_{2} p_{3} \cdots p_{n-2} p_{n}\right)\right) \\
& +\cdots+\left(p_{3} \cdots p_{n-1} p_{n}\right)^{(1-a)} \cos \left(b \ln \left(p_{3} \cdots p_{n-1} p_{n}\right)\right) \\
& +\left(p_{2} p_{3} \cdots p_{n-1} p_{n}\right)^{(1-a)} \cos \left(b \ln \left(p_{2} p_{3} \cdots p_{n-1} p_{n}\right)\right)=0 \\
& \left(p_{2} p_{3} \cdots p_{n-1}\right)^{(1-a)} \cos \left(\frac{\pi}{2}-b \ln \left(p_{2} p_{3} \cdots p_{n-1}\right)\right) \\
& +\left(p_{2} p_{3} \cdots p_{n-2} p_{n}\right)^{(1-a)} \cos \left(\frac{\pi}{2}-b \ln \left(p_{2} p_{3} \cdots p_{n-2} p_{n}\right)\right) \\
& +\cdots+\left(p_{3} \cdots p_{n-1} p_{n}\right)^{(1-a)} \cos \left(\frac{\pi}{2}-b \ln \left(p_{3} \cdots p_{n-1} p_{n}\right)\right) \\
& +\left(p_{2} p_{3} \cdots p_{n-1} p_{n}\right)^{(1-a)} \cos \left(\frac{\pi}{2}-b \ln \left(p_{2} p_{3} \cdots p_{n-1} p_{n}\right)\right)=0
\end{aligned}
$$

According to the theory of infinite series, when the number of items tends to infinite, to make Equations (76) and (77) be tenable simultaneously, besides $a \rightarrow \infty$, the only way is to let the corresponding terms are equal to each other, we have

$$
\begin{aligned}
& b=\frac{\pi}{4 \ln \left(p_{2} p_{3} \cdots p_{n-1}\right)}, \quad b=\frac{\pi}{4 \ln \left(p_{2} p_{3} \cdots p_{n-2} p_{n}\right)} \\
& b=\frac{\pi}{4 \ln \left(p_{3} \cdots p_{n-1} p_{n}\right)}, \quad b=\frac{\pi}{4 \ln \left(p_{2} p_{3} \cdots p_{n-1} p_{n}\right)}
\end{aligned}
$$

And so on. However, Equation (78) are impossible, so Equations (76) and (77) can not be equal to zeros simultaneously. The summation form of Zeta function $\zeta(1-s)$ can not be zero, and so do for $\zeta\left(s^{\prime}\right)$ to let $s^{\prime} \rightarrow 1-s$. Then the proof of Theorem 3 is finished.

The result above has nothing to do with the value of $a$, as long as $a<\infty$, no matter $a>1$ or $a \leq 1$. If take $b=0$, i.e., on the real axis, Equation (51) becomes zero but Equation (50)is not equal to zero. We have

$$
\zeta(1-a)=\zeta_{1}(1-a)=1+2^{a-1}+3^{a-1}+\cdots+n^{a-1}+\cdots \neq 0
$$

If taking $a=1 / 2$, we have $1-a=1 / 2$. It is proved in [1] that only in this case, the two sides of Equation (2) can be equal to each other. But the result is infinite, rather than zero, so it is meaningless.

$$
\zeta(1 / 2)=1+\frac{1}{\sqrt{2}}+\frac{1}{\sqrt{3}}+\cdots+\frac{1}{\sqrt{n}}+\cdots \rightarrow \infty
$$

According to the discussion above, the Zeta function equations Equation (4) and Equation (5) has no non-trivial zeros. 
Up to now, we have fully explained the problem of Riemann hypothesis, and obtained the following Theorem 4 .

Theorem 4: The Riemann Zeta function Equations (4) and (5) have no non-trivial zeros on whole complex plane. The Riemann hypothesis does not hold.

\section{Conclusions}

The Riemann Zeta function equation has two forms. They are considered with the same non-trivial zeros. The Riemann hypothesis claims that all non-trivial zeros were located on the critical line $\operatorname{Re}(s)=1 / 2$ of complex plane, but it can not be proved up to now.

In the author previous paper "The inconsistency problem of Riemann Zeta function equation", it was revealed that there were four basic mistakes in the Riemann's original paper proposed in 1895. The Riemann Zeta function equation did not hold and the Riemann hypothesis was meaningless.

In this paper, we suppose that the Riemann Zeta function equations still hold. By separating the Zeta function equation into real part and imaginary part completely, it is proved that the Riemann Zeta function equations have no non-trivial zeros. The summation form of Zeta function itself also has no zeros. The Riemann hypothesis is proved untenable again from another angle.

\section{Conflicts of Interest}

The author declares no conflicts of interest regarding the publication of this paper.

\section{References}

[1] Mei, X.C. (2019) The Inconsistency Problem of Riemann Zeta Function Equation. Mathematics Letters, 5, 13-22.

http://www.sciencepublishinggroup.com/journal/paperinfo?journalid=348\&doi=10. 11648/j.ml.20190502.11

[2] Riemann, G.F.B. (1859) Uber die Anzabl der Primahlem unter einer gegebenen Grosse. Monatsberichte der Berliner Akademine, 2, 671-680.

[3] Petersen, B.E. (1996) Riemann Zeta Function. https://pan.baidu.com/s/1geQsZxL

[4] Neukirch, J. (1999) Algebraic Number Theory. Springer, Berlin, Heidelberg. (The original German Edition Was Published in 1992 under the Title Algebraische Zahlentheorie). https://doi.org/10.1007/978-3-662-03983-0

[5] Guo, D.R. (1965) The Method of Mathematics and Physics. People's Education Press, Beijing, 109.

[6] Gourdon, X. (2004) The $10^{13}$ First Zeros of the Riemann Zeta Function, and Zeros Computation at Very Large Height. http://pdfs.semanticscholar.org/6eff/62ff5d98e8ad2ad8757c0faf4bac87546f27.pdf

[7] Ru, C.H. (2016) The Riemann Hypothesis. Qianghua University Publishing Company, Beijing, p. 61, 52, 192. 